\title{
Effects of mandibular advancement on airway curvature and obstructive sleep apnoea severity
}

\author{
S. Tsuiki*,\#, A.A. Lowe*, F.R. Almeida*, N. Kawahata", J.A. Fleetham ${ }^{+}$
}

Effects of mandibular advancement on airway curvature and obstructive sleep apnoea severity. S. Tsuiki, A.A. Lowe, F.R. Almeida, N. Kawahata, J.A. Fleetham. (C)RS Journals Ltd 2004.

ABSTRACT: In a curved tube, the amount of airflow appears to be influenced by the amount of curvature. The purpose of this study was to investigate changes in obstructive sleep apnoea (OSA) severity and awake velopharyngeal curvature in response to an anteriorly titrated mandibular position in 20 male OSA patients.

Baseline supine cephalometry was obtained before the initial insertion of a titratable oral appliance and follow-up supine cephalometry was undertaken after titration of the mandibular position with the appliance in place.

The mean apnoea/hypopnea index (AHI) before treatment $\left(31.6 \pm 13.0\right.$ events $\left.\cdot h^{-1}\right)$ was significantly reduced $\left(9.8 \pm 7.4\right.$ events $\left.\cdot h^{-1}\right)$ after titration of the mandibular position in all 20 patients. There was a significant increase in the anteroposterior calibre and the radius of the curvature of the anterior wall of the velopharynx in $14 \operatorname{good}$ responders who exhibited an AHI reduction to $\leqslant 15$. Similar observations were not found in six poor responders.

To conclude, an anteriorly titrated mandibular position reduced obstructive sleep apnoea severity, enlarged the velopharynx and diminished the curvature of the anterior velopharyngeal wall in good responders. It is proposed that this change in the upper airway curvature associated with mandibular advancement may effect obstructive sleep apnoea severity through its effect on airflow dynamics.

Eur Respir J 2004; 23: 263-268.
*Dept of Oral Health Sciences, Faculty of Dentistry, and ${ }^{+}$Division of Respiratory Medicine, Faculty of Medicine, The University of British Columbia, Vancouver, BC, Canada, and ${ }^{\#}$ Maxillofacial Orthognathics, Graduate School, Tokyo Medical and Dental University, Tokyo, and Dept of Oral and Maxillofacial Prosthodontics, Field of Oral and Maxillofacial Rehabilitation, Kagoshima University Graduate School of Medical and Dental Sciences, Kagoshima, Japan.

Correspondence: A.A. Lowe, Dept of Oral Health Sciences, Faculty of Dentistry, The University of British Columbia, 2199 Wesbrook Mall, Vancouver BC, Canada V6T 1Z3. Fax: 16048223562

E-mail: alowe@interchange.ubc.ca

Keywords: Airway curvature, mandibular advancement, obstructive sleep apnoea, titratable oral appliance

Received: October 162002

Accepted after revision: September 22003

Part of this study was supported by Grants-inAid for Scientific Research Projects (15659469) from the Japanese Ministry of Education, Culture. F.R. Almeida was supported by a scholarship from $\mathrm{CNP}_{9}$-Brazil, Brazil.
The fluid flow through curved tubes in human organs such as the aortic arch and the coronal arteries are of interest physiologically in the study of cardiovascular systems, since an increase in the curvature induces pressure losses as well as higher resistance in the same region $[1,2]$. The upper airway (UA) has characteristics similar to those of the curved vessels in the cardiovascular system in that the airstream is curved from the nasal cavity to the hypopharynx at the level of the velopharynx. Nevertheless, an interaction between the curvature of the velopharynx and the pathogenesis of obstructive sleep apnoea (OSA) remains unknown.

Recent developments in mandibular advancement oral appliances (OAs) have provided a better quality of life not only for patients with snoring and mild-to-moderate OSA, but also for severe OSA patients who could not tolerate nasal continuous positive airway pressure (nCPAP) [3, 4]. An anterior titration of the mandibular position using OAs has been reported to increase the anteroposterior diameter [5] and cross-sectional area [6] of the velopharynx, resulting in a reduction of OSA severity. In addition to such anatomical changes, it was reported that advancement of the mandible decreased nasal resistance and facilitated nasal breathing [7, 8]. These reports indicate a close relationship between morphological alteration and improvement of airflow dynamics in response to mandibular protrusion. If the anterior titration of the mandibular position decreased the curvature of the velopharynx, it may assist in improving airflow dynamics.

It was hypothesised that an anteriorly titrated mandibular position would diminish the curvature of the UA and lead to a reduction in OSA severity. Accordingly, the objectives of this study were to evaluate changes in the velopharyngeal curvature and OSA severity following a forward titration of the mandibular position using an $\mathrm{OA}$, and to speculate as to the clinical significance of these changes in OSA treatment.

\section{Methods}

\section{Subjects}

A total of 20 male OSA patients (apnoea/hypopnoea index (AHI) $>15$ events $\cdot \mathrm{h}^{-1}$, range $\left.16.8-57.8\right)$ were recruited for this study. Ten patients exhibited moderate OSA $(15<$ AHI $\leqslant 30$ events $\cdot \mathrm{h}^{-1}$, range $\left.16.8-28.6\right)$ whereas the remaining 10 severe OSA patients (AHI $>30$ events $\cdot \mathrm{h}^{-1}$, range 32.2-57.3) 
were nCPAP failures. Patients who had undergone uvulopalatopharyngoplasty were not included. The mean \pm SD age of the patients was $44.7 \pm 10.2$ yrs and the mean \pm SD body mass index $(\mathrm{BMI})$ was $27.8 \pm 4.9 \mathrm{~kg} \cdot \mathrm{m}^{-2}$. Each patient provided written informed consent. The study protocol was approved by the Clinical Screening Committee for Research and Other Studies Involving Human Subjects at the University of British Columbia.

\section{Diagnostic polysomnography}

All patients were diagnosed with OSA based on polysomnographic sleep studies obtained at the Sleep Disorders Clinic of the Vancouver Hospital and Health Sciences Centre [6]. A second overnight polysomnographic study was undertaken with the titratable OA in place $[4,6]$.

\section{Titratable oral appliance}

A two-piece titratable mandibular advancement OA (Klearway ${ }^{\text {IM}}$; Space Maintainers Laboratories Canada Ltd, Vancouver, BC, Canada) was used to titrate the mandibular position forward [4-6] (fig. 1). Details of the Klearway ${ }^{\text {TM }}$ appliance including fabrication and adjustment have been described elsewhere $[4,6]$.

\section{Supine cephalometry}

Baseline supine cephalometry was obtained before initial insertion of the OA and follow-up supine cephalometry was undertaken after titration with the $\mathrm{OA}$ in place by an established method [5, 9, 10]. Two reference lines (fig. 2) were constructed to perform the velopharyngeal measurements: 1) So line (reference line that goes through the anterior nasal spine and the posterior nasal spine); 2) $\mathrm{N} \perp \mathrm{S} 0$ line (reference line that is perpendicular to the So line and passes through the nasion). The superior level of the velopharynx was defined as the backward extended So line, and the inferior margin of the velopharynx was a line drawn through the tip of

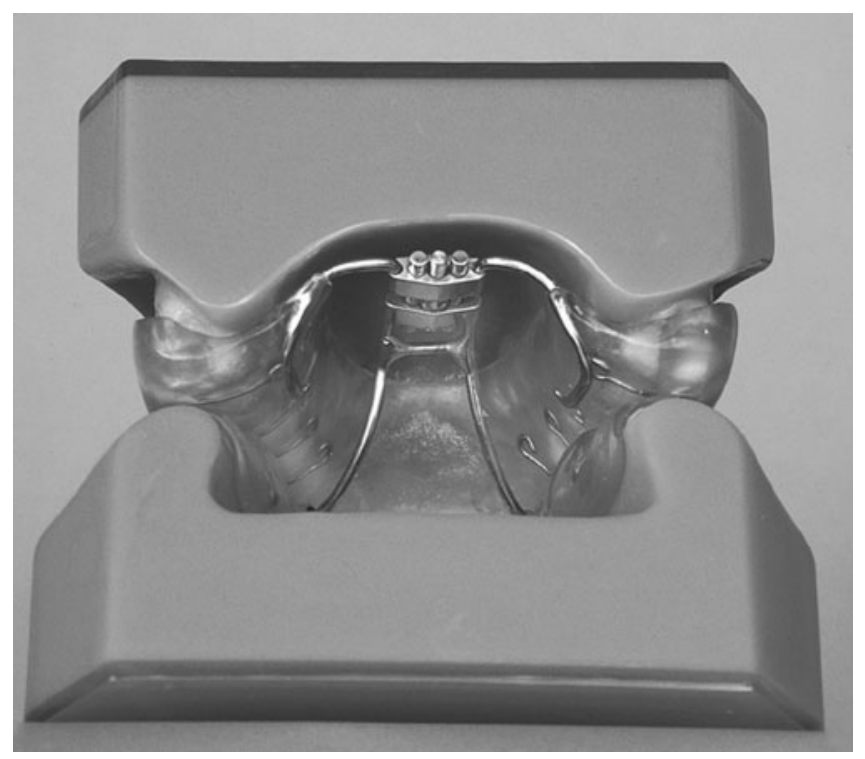

Fig. 1.- Klearway ${ }^{\mathrm{TM}}$ titratable oral appliance. The adjustable screw mechanism, which is located in the palatal arch, permits $11 \mathrm{~mm}$ of mandibular protrusion in 44 increments of $0.25 \mathrm{~mm}$ and also allows vertical and horizontal jaw movement. the soft palate parallel to the S0 line. The velopharynx was divided into five slices $(\mathrm{S} 0-\mathrm{S} 4)$ of equal distance by lines parallel to the S0 line. Three landmark points (P1, P2 and P3) on the anterior velopharyngeal wall were defined to assume the superior part of the anterior velopharyngeal wall as a part of a circle (fig. 2). A circle determined by the three points was constructed and its radius of curvature was calculated using custom-made software. All of the cephalometric analyses were performed by the same orthodontist before the follow-up polysomnographic study. The value of method errors for the cephalometric analyses (range $0.25-0.63 \mathrm{~mm}$ ) was consistent with those in previous studies $[10,11]$.

\section{Titration protocol}

A schematic representation of this study is provided in figure 3. The OA was inserted at $67 \%$ of the maximum protruded position after completing baseline polysomnography and supine cephalometry without the OA in place. Once patients were accustomed to wearing the OA without any discomfort, they were instructed to start advancing the mandible with gradual increments of 0.25 or $0.50 \mathrm{~mm}$ per week. The amount of mandibular advancement was checked at every visit. The patients were also advised to stop advancing the mandible until the next visit if temporomandibular joint or jaw muscle discomfort occurred. When the patient or bed partner reported a cessation of snoring and a resolution of symptoms, further advancement was not performed and it was considered titrated. The screw of the OA was tied off after confirming that the patient felt the titrated mandibular position was comfortable $[4,6]$. Follow-up polysomnography and supine cephalometry were completed with the OA in place.

\section{Statistical analysis}

The normality of the distribution of the data was checked before statistical analysis [12]. Data are presented as mean \pm SD and paired t-tests were obtained to compare the differences in each variable between baseline and after titration values unless otherwise stated. On the basis of after titration AHI values, the 20 patients were divided into good responders where the AHI was reduced to $\leqslant 15$ events $\cdot h^{-1}$ and poor responders where the AHI was $>15$ events $\cdot h^{-1}$. An unpaired t-test was used to compare the polysomnographic and cephalometric variables between the two groups unless otherwise stated. A p-value of $<0.05$ was considered to be statistically significant.

\section{Results}

Sixteen of the 20 patients reported a cessation of snoring and reduction in daytime sleepiness whereas residual snoring was observed in four patients, although these patients reported a reduction in OSA symptoms. The mean per cent amount of additional advancement beyond $67 \%$ of maximum mandibular protrusion during the titration period $(140 \pm 87$ days) was $16.2 \pm 8.6 \%$.

In all 20 patients, both the mean apnoea index (AI) and AHI before treatment were significantly reduced after titration of the mandibular position (AI $7.3 \pm 9.1$ to $1.5 \pm 2.5$ events $\cdot \mathrm{h}^{-1}$, $\mathrm{p}<0.01$; AHI $31.6 \pm 13.0$ to $9.8 \pm 7.4$ events $\left.\cdot \mathrm{h}^{-1}, \mathrm{p}<0.001\right)$. The minimum arterial oxygen saturation $\left(\mathrm{Sa}_{\mathrm{a}} \mathrm{O}_{2}\right)$ also improved significantly $\left(77.2 \pm 11.3 \%\right.$ to $83.6 \pm 7.2$ events $\left.\cdot h^{-1}, \mathrm{p}<0.01\right)$. No statistically significant change was observed in BMI during the study $\left(27.8 \pm 4.9\right.$ to $\left.27.6 \pm 4.5 \mathrm{~kg} \cdot \mathrm{m}^{-2}, \mathrm{p}>0.05\right)$. On the basis of after titration AHI values, the 20 patients were divided into 

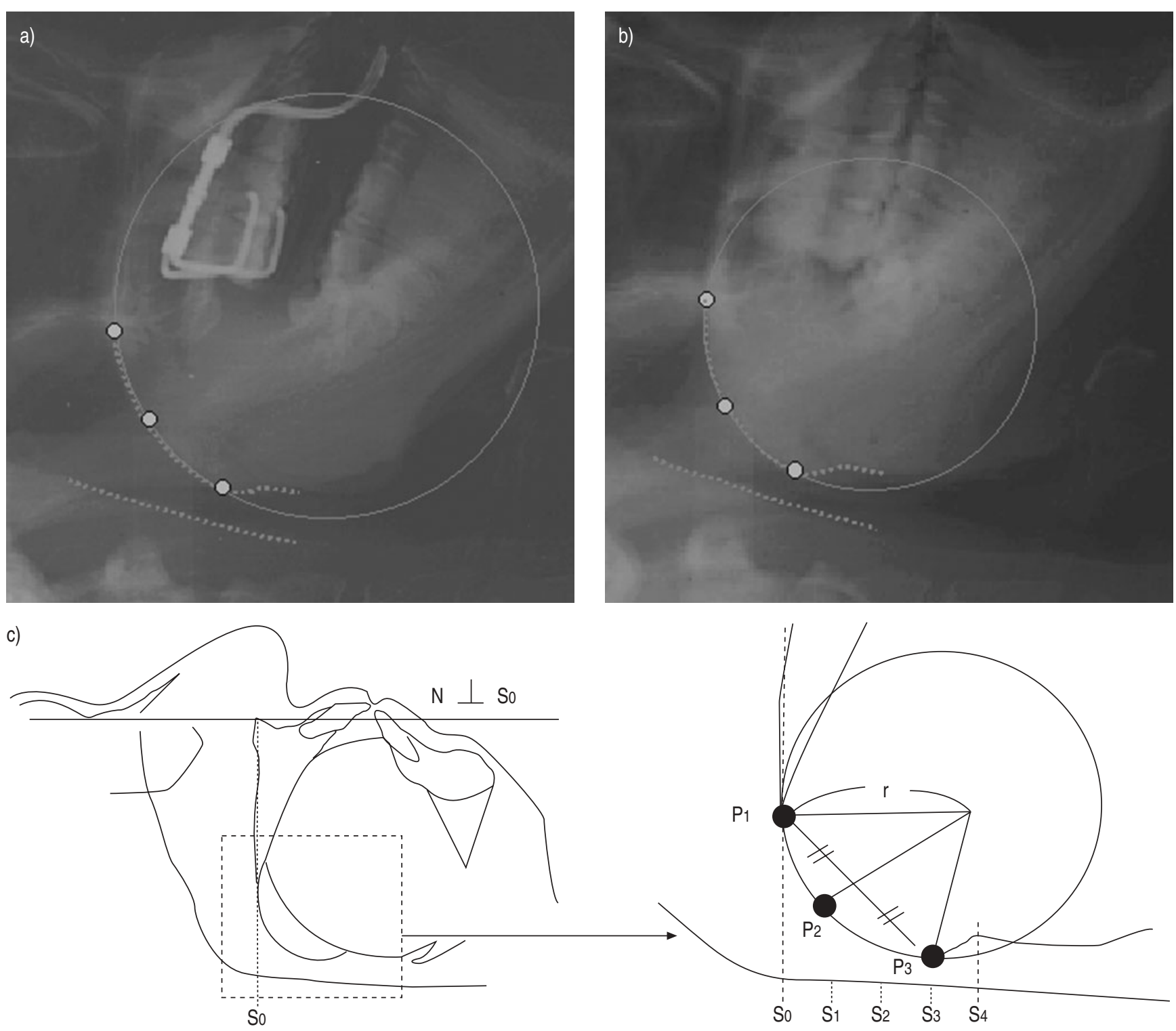

Fig. 2. - Soft tissue movements a) with and b) without the titratable oral appliance and c) diagramatic representation of airway measurements. S0 line: reference line that goes through the anterior nasal spine and the posterior nasal spine; $\mathrm{N} \perp \mathrm{S}$ o line: reference line that is perpendicular to the So line and passes through nasion; $\mathrm{P}_{1}$ : intersection between $\mathrm{S}_{0}$ and the anterior velopharyngeal wall; $\mathrm{P}_{3}$ : intersection between the anterior velopharyngeal wall and a line parallel to the So line that goes through the narrowest part of the velopharynx; P2: intersection between the anterior velopharyngeal wall and a line perpendicular to $\mathrm{P}_{1}-\mathrm{P}_{3}$ line that divides $\mathrm{P}_{1}-\mathrm{P}_{3}$ into two equal segments; r: radius of the circle that is determined from $\mathrm{P}_{1}, \mathrm{P}_{2}$ and $\mathrm{P}_{3}$; $\mathrm{S}_{1}-\mathrm{S}_{4}$ : anteroposterior distance of velopharynx at each slice level.

good responders $(n=14)$ where the AHI was reduced to $\leqslant 15$ events $\cdot h^{-1}$ and poor responders $(n=6)$ where the AHI was reduced to $>15$ events $\cdot h^{-1}$ (fig. 4). There was no significant difference in the total amount of mandibular advancement between the good responders $(10.5 \pm 1.5 \mathrm{~mm})$ and the poor responderes $(10.5 \pm 1.4 \mathrm{~mm}, \mathrm{p}=0.88)$.

The effects of the anterior titration of the mandibular position on respiratory and sleep variables in the two groups are summarised in table 1 . In good responders, both the mean $\mathrm{AI}$ and AHI before treatment were significantly reduced after titration of the mandibular position $(\mathrm{p}<0.001)$. The minimum $\mathrm{Sa}, \mathrm{O}_{2}$ also significantly improved $(\mathrm{p}<0.05)$. In addition, Stage 1 sleep and the arousal index significantly decreased (Stage 1 sleep $\mathrm{p}<0.05$; arousal index $\mathrm{p}<0.001)$. Similar significant observations were not seen in the poor responders.

Table 2 shows the effects of anterior titration of the mandibular position on cephalometric variables in both good responders and poor responders. Following the titration of the OA, a significant increase in the anteroposterior distance was observed at $\mathrm{S} 0(\mathrm{p}<0.05), \mathrm{S} 1(\mathrm{p}<0.01)$ and $\mathrm{S} 2(\mathrm{p}<0.01)$ in good responders. These observations were seen in response to a forward repositioning of the anterior velopharyngeal wall (fig. 5). Moreover, there was a significant increase in the radius of curvature of the anterior wall of the velopharynx $(\mathrm{p}<0.05$; table 2). Although there was a slight enlargement tendency at $\mathrm{S} 2(\mathrm{p}=0.052)$, no significant difference was found in either the anteroposterior dimension or the radius of curvature in poor responders.

\section{Discussion}

The results of this study demonstrated a significant reduction in OSA severity, enlargement of the velopharynx 


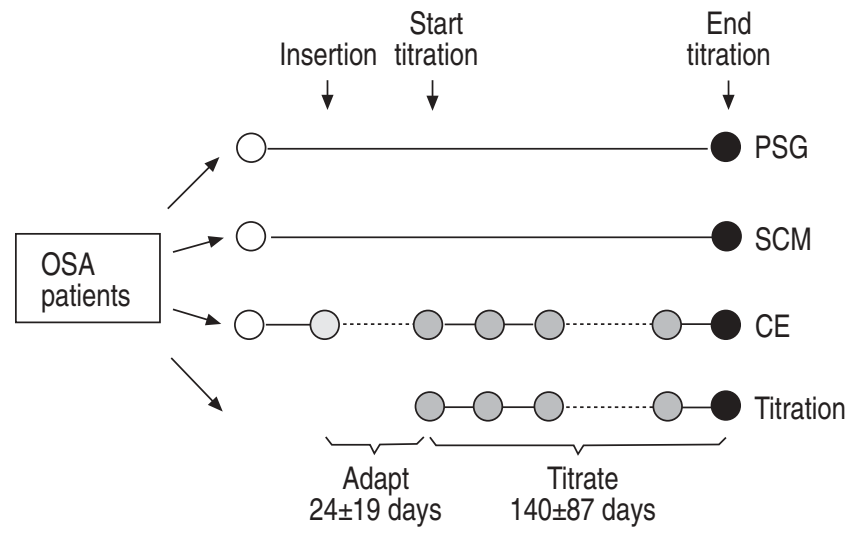

Fig. 3.-Schematic illustration of the study protocol. After an initial baseline evaluation $(O)$ and an adaptation period with the titratable oral appliance, the mandible was gradually advanced in $0.25-\mathrm{mm}$ increments. Polysomnography and supine cephalometry were taken at the baseline visit and again after titration. The adjustment screw was tied off when symptoms of obstructive sleep apnoea (OSA) disappeared, and a follow-up evaluation was performed (๑). PSG: polysomnography; SCM: supine cephalometry; CE: clinical evaluation.

and changes in the radius of curvature of the anterior velopharyngeal wall following insertion of the titratable OA in good responders. Although the functional properties of the UA including the relationship between mandibular advancement and UA muscle activity [13] in OSA patients are still to be tested, it is no longer in question that mandibular advancement improves the $\mathrm{AHI}$ and the minimum $\mathrm{Sa}_{\mathrm{a}} \mathrm{O}_{2}$

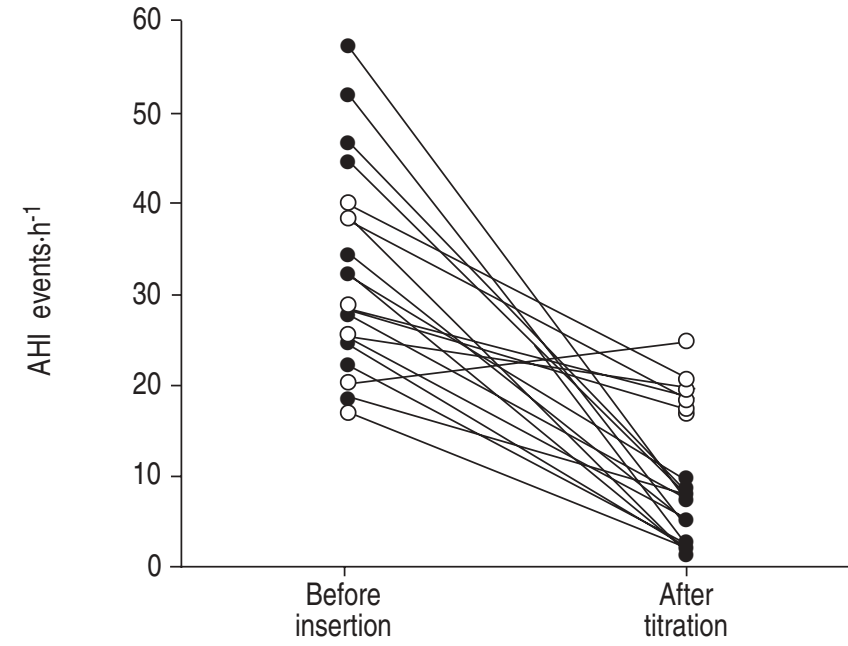

Fig. 4. - Apnoea/hypopnoea index (AHI) before titration and after titration of the titratable oral appliance. 14 good responders (AHI was reduced to $\leqslant 15$ events $\cdot h^{-1}$ ); $\bigcirc$ : six poor responders (AHI was reduced to $>15$ events $\cdot h^{-1}$ ).

and increases the calibre, cross-sectional area, and possibly, the volume of the UA [4, 6, 14-17]. The results of this study are consistent with previous reports obtained from titration protocols of OAs [4, 6, 17].

Interestingly, the anteriorly titrated mandibular position lengthened the radius of curvature of the anterior velopharyngeal wall in good responders (fig. 6). Since the anterior

Table 1.-Effects of anterior titration of mandibular position on respiratory and sleep variables

\begin{tabular}{|c|c|c|c|c|}
\hline \multirow[t]{2}{*}{ Characteristics } & \multicolumn{2}{|c|}{ Good responders } & \multicolumn{2}{|c|}{ Poor responders } \\
\hline & Before insertion & After titration & Before insertion & After titration \\
\hline Age yrs & $43.3 \pm 10.8$ & $43.8 \pm 10.1 * * *$ & $48.4 \pm 7.7$ & $48.8 \pm 7.7 * * *$ \\
\hline $\mathrm{BMI} \mathrm{kg} \cdot \mathrm{m}^{-2}$ & $26.7 \pm 4.0$ & $26.3 \pm 3.4$ & $31.1 \pm 6.0$ & $30.8 \pm 5.8$ \\
\hline AI events $\cdot h^{-1}$ & $9.6 \pm 10.6$ & $0.7 \pm 1.3^{* *,+}$ & $3.1 \pm 2.0$ & $3.1 \pm 3.5$ \\
\hline AHI events $\cdot h^{-1}$ & $33.8 \pm 13.0$ & $5.2 \pm 3.0 * * *, \S$ & $28.5 \pm 15.1$ & $18.2 \pm 5.0$ \\
\hline Minimum $\mathrm{Sa}_{\mathrm{a}, \mathrm{O}_{2}} \%$ & $78.3 \pm 8.9$ & $85.0 \pm 6.9^{*}$ & $75.0 \pm 15.4$ & $79.4 \pm 6.4$ \\
\hline Total sleep time min & $350(308-398)$ & $336(256-399)$ & $331(303-394)$ & $362(353-420)$ \\
\hline Stage 1 sleep $\%$ & $19.7(8.6-28.5)$ & $11.8(6.6-17.9)^{*, f}$ & $15.2(6.0-15.3)$ & $9.4(8.7-10.8)$ \\
\hline Stage 2 sleep $\%$ & $54.5(41.2-66.2)$ & $57.3(53.6-69.2)$ & $65.0(62.5-68.3)$ & $65.9(64.8-69.5)$ \\
\hline SWS \% & $8.7(0.4-14.7)$ & $2.5(1.7-9.3)$ & $0.1(0.0-3.0)$ & $3.0(2.6-7.7)$ \\
\hline REM sleep \% & $16.5(14.7-18.5)$ & $18.9(15.0-21.6)$ & $16.6(14.0-20.5)$ & $21.2(13.9-22.4)$ \\
\hline Arousal index events $\cdot \mathrm{h}^{-1}$ & $25.9(12.0-30.7)$ & $13.3(4.5-17.2)^{* *, f}$ & $21.3(13.3-26.0)$ & $14.0(11.1-21.6)$ \\
\hline
\end{tabular}

Data are presented as mean \pm SD or median (interquartile range) unless otherwise stated; BMI: body mass index; AI: apnoea index; AHI: apnoea/ hypopnoea index; $\mathrm{Sa}, \mathrm{O}_{2}$ : arterial oxygen saturation; SWS: slow-wave sleep; REM: rapid eye movement. ${ }^{\#}: \mathrm{n}=14 ; \boldsymbol{\tau}^{:}: \mathrm{n}=6$; $*: \mathrm{p}<0.05$ versus before insertion; ${ }^{* *}: \mathrm{p}<0.01$ versus before insertion; ${ }^{* * *}: \mathrm{p}<0.001$ versus before insertion; ${ }^{+}: \mathrm{p}<0.05$ versus poor responder; ${ }^{\$}$ : $\mathrm{p}<0.001$ versus poor responder; ${ }^{f}$ : Wilcoxon signed-rank test.

Table 2. - Effects of anterior titration of mandibular position on cephalometric variables

\begin{tabular}{|c|c|c|c|c|}
\hline & \multicolumn{2}{|c|}{ Good responders $^{\#}$} & \multicolumn{2}{|c|}{ Poor responders } \\
\hline & Before insertion & After titration & Before insertion & After titration \\
\hline r mm & $68.6 \pm 28.2$ & $80.7 \pm 33.3^{*}$ & $62.8 \pm 14.0$ & $70.8 \pm 14.8$ \\
\hline S0 mm & $19.3 \pm 4.5$ & $20.9 \pm 4.9^{*}$ & $23.4 \pm 3.9$ & $23.4 \pm 3.5$ \\
\hline $\mathrm{S} 1 \mathrm{~mm}$ & $11.2 \pm 3.4$ & $12.9 \pm 4.1 * *$ & $9.3 \pm 4.5$ & $12.0 \pm 2.7$ \\
\hline $\mathrm{S} 2 \mathrm{~mm}$ & $7.2 \pm 3.2$ & $8.4 \pm 2.8 * *$ & $6.0 \pm 3.5$ & $7.7 \pm 2.1$ \\
\hline $\mathrm{S} 3 \mathrm{~mm}$ & $5.4 \pm 2.3$ & $6.1 \pm 3.4$ & $6.8 \pm 3.4$ & $7.9 \pm 5.8$ \\
\hline $\mathrm{S} 4 \mathrm{~mm}$ & $6.3 \pm 1.8$ & $7.0 \pm 3.5$ & $7.1 \pm 2.8$ & $8.8 \pm 2.8$ \\
\hline
\end{tabular}

Values are presented as mean \pm SD unless otherwise stated. r: radius of curvature of anterior velopharyngeal wall; S0-S4: anteroposterior distance of velopharynx at each slice level. ${ }^{\#}: \mathrm{n}=14 ;{ }^{\bullet}: \mathrm{n}=6 ; *$ : $\mathrm{p}<0.05$ versus before insertion; **: $\mathrm{p}<0.01$ versus before insertion. 


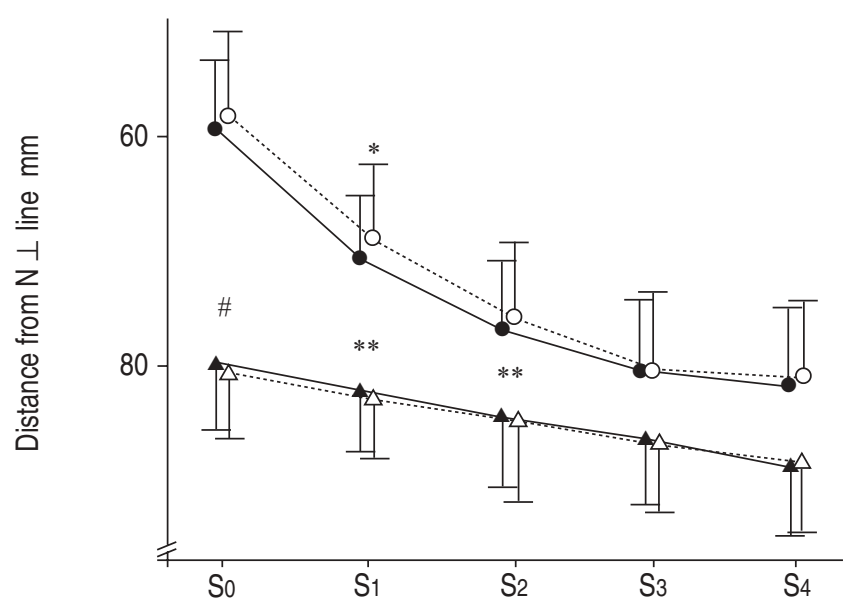

Fig. 5. - Slice level changes in the anteroposterior dimension of the velopharynx in response to mandibular advancement in 14 good responders. - : anterior wall before insertion; $\bigcirc$ : anterior wall after titration; $\boldsymbol{\Delta}$ : posterior wall before insertion; $\triangle$ : posterior wall after titration. *: $\mathrm{p}<0.05$ anteroposterior position before insertion versus after titration at the point; $: \mathrm{p}<0.05$ anteroposterior distance before insertion versus after titration at the slice level; **: $\mathrm{p}<0.01$ anteroposterior distance before insertion versus after titration at the slice level.

velopharyngeal wall corresponds to the posterior border of the soft palate, lengthening of this structure could contribute to the increase of the radius.

RYAN et al. [6] speculated that traction on the palatoglossus and palatopharyngeus muscles may increase anteroposterior and lateral dimensions of the velopharynx. The radius of curvature of the tube is a key element in determining the resistance and pressure distribution [1,2]. The flow in a curved pipe is considerably more complex than that in a straight conduit since secondary flows, which are induced by transverse velocities, appear whenever fluid flows in a curved pipe [1]. The secondary flow gives rise to asymmetrical forces with a low pressure region [2]. Since the basic principle is the same between gases and fluids, the present authors speculated that a reduction in UA curvature acts to diminish the resistance and the pressure drop in the UA. The anterior

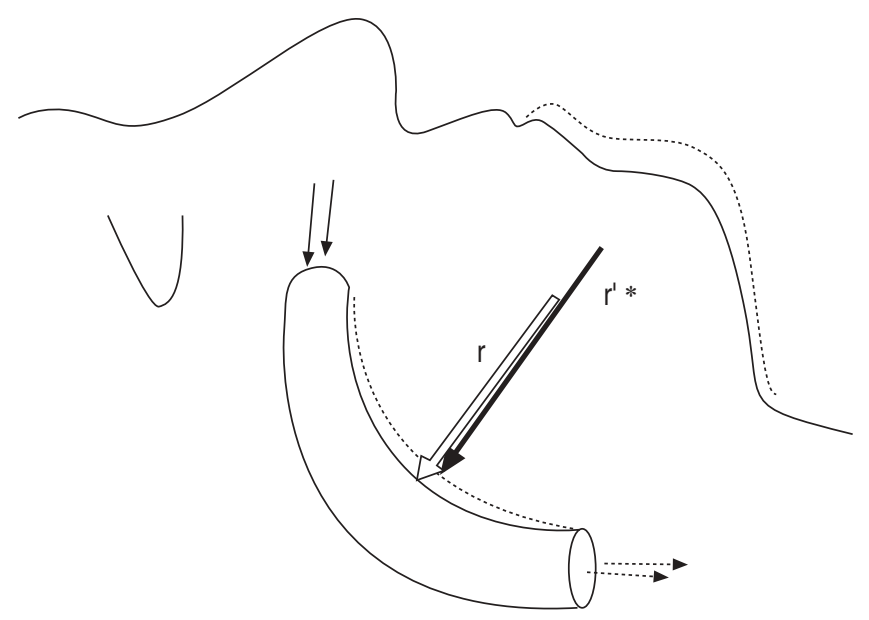

Fig. 6. - Schematic illustration to show the possible effects of mandibular advancement on the anterior velopharyngeal wall in good responders. Open and filled arrows denote the radius of curvature of the anterior velopharyngeal wall before $(-)$ initial insertion $(r$ $68.6 \pm 28.2 \mathrm{~mm})$ and after titration $(\cdots \cdots \cdots)\left(\mathrm{r}^{\prime} 80.7 \pm 33.3 \mathrm{~mm}\right)$ of the oral appliance, respectively. Dashed arrows indicate the direction of airflow. Note that the anteriorly titrated mandibular position decreases the curvature of the anterior velopharyngeal wall. *: $\mathrm{p}<0.05$ versus before insertion. velopharyngeal wall was evaluated and regarded as a part of a circle to make the analysis simple, since the posterior velopharyngeal wall is more rigid and less influenced by changes in regional structure induced by external perturbations [18]. The present study also demonstrated that the posterior wall of the velopharynx remains unchanged in response to mandibular protrusion (fig. 3).

A robust defitition in terms of AHI cut-off criteria was used [19]. A complete response (follow-up AHI of <5) was observed in six patients and a partial response (follow-up AHI of $\geqslant 5$ but $>50 \%$ reduction in AHI) was seen in eight patients of the 14 good responders. Good responders to the titratable $\mathrm{OA}$ in the present study still demonstrated either a complete response or partial response in 14 of 20 patients, whereas six patients were regarded as poor responders. These results should be reconfirmed in a future study with a larger sample size.

Previous studies have confirmed that the velopharynx is the most common site of narrowing in the UA of OSA patients [20-22]. Therefore, the fact that mandibular advancement mechanically enlarges and improves velopharyngeal patency seems to be a reasonable concept $[4,21]$. As long as the same volume of tidal air passes through a tube during one inspiratory phase, acceleration of airflow at the narrowed site implies a gain in kinetic energy (i.e. velocity) and corresponding loss of potential energy (i.e. pressure). The loss of the potential energy indicates a decrease in pressure at the site of UA narrowing, which will tend to collapse it further [9, 23]. It has been demonstrated in vitro that an $8-\mathrm{mm}$ protrusion of the mandible results in an enlargement of the cross-sectional area of the narrowed site, a reduction of the velocity of airflow, and a decrease in the pressure drop in the UA [24]. The enlargement of the velopharynx caused by a repositioning of the soft palate in this study may bring about the same results obtained from the in vitro study [24]. Collectively, the authors speculate that a combination of some or all of the factors described above, including the radius of curvature of the velopharynx, may provide decreased UA vulnerability in OA therapy. It was reported that both nCPAP and OAs enlarge the transverse as well as the anteroposterior dimension of the UA [25]; however, changes in the velopharyngeal curvature caused by mandibular advancement may be a unique mode of action for OA therapy.

There are some significant limitations to this study and to OA therapy overall. The cephalometric data in the awake state significantly limits extrapolation of the findings to the sleeping state. However, a recent study in control subjects revealed that mandibular advancement during sleep still enlarged the midlevel of the velopharynx, as well as during wakefulness [5], due to a repositioning of the anterior velopharyngeal wall [26]. Thus, it is speculated that mandibular advancement would provide the same effects on asleep velopharyngeal curvature although the amount of changes in the radius seems to be different between the awake and asleep status. The authors accept that this study with twodimensional cephalometry has known limitations to measure the transverse dimension [25], cross-sectional shape [27] and volume [28] of the UA. However, as long as data are collected following a standardised method $[5,10]$, the superimposition of cephalometric tracings appears to be useful enough for the analysis of UA curvature in the sagittal dimension. Since the titration period $(140 \pm 87$ days) of the OA in order to avoid known side-effects [4] is obviously longer than with nCPAP, where titration of nasal pressure is typically achieved in one night, it is acknowledged that this longer time period is a limitation of OA therapy especially when a rapid therapeutic response is required.

The reason(s) why six of 20 patients did not show a good response in terms of respiratory and cephalometric variables 
is unknown. A relatively small sample size limits the answers to such questions. Better methods to predict the efficacy of $\mathrm{OA}$ and the correlation between change in AHI and the radius of curvature are needed. However, the effectiveness of OA therapy is supported by other aspects in that patients strongly prefer OAs to nCPAP [29], and they are relatively easy to use [4]. Finally, the interaction between airflow dynamics and velopharyngeal curvature should be investigated further in a larger sample size.

To conclude, anterior titration of the mandibular position improved obstructive sleep apnoea severity and resulted in an enlarged upper airway. Moreover, it changed the curvature of the anterior velopharyngeal wall, which may change airflow dynamics during inspiration. The authors believe that the results of this study could be a starting point to provide new insight into the relationship between curvature of the upper airway and airflow dynamics. Two and three-dimensional in vitro modelling of airflow in humans [24, 30] may be one possible method to further evaluate the effectiveness of oral appliances in the treatment of obstructive sleep apnoea.

Acknowledgement. The authors would like to thank C.I.R. Almeida, B. Gick and M. Takekawa for their helpful suggestions. The authors are also grateful to I. Ellis for editorial assistance with this manuscript. The Klearway $^{\mathrm{TM}}$ appliance was invented by one of the authors. International patents have been obtained by The University of British Columbia and specific licensees are assigned the rights to manufacture and distribute the appliance worldwide.

\section{References}

1. Berger SA, Talbot L, Yao LS. Flow in curved pipes. Ann Rev Fluid Mech 1983; 15: 461-512.

2. Sarin VB. Flow of an elastico-viscous liquid in a curved pipe of slowly varying curvature. Int J Biomed Comput 1993; 32: 135-149.

3. Schmidt-Nowara W. Recent developments in oral appliance therapy of sleep disordered breathing. Sleep Breath 1999; 3: 103-106.

4. Lowe AA. Oral appliances for sleep breathing disorders. In: Kryger MH, Roth T, Dement WC, eds. Principles and Practice of Sleep Medicine. 3rd Edn. Philadelphia, WB Saunders, 2000; pp. 929-939.

5. Tsuiki S, Hiyama S, Ono T, et al. Effects of a titratable oral appliance on supine airway size in awake non-apneic individuals. Sleep 2001; 24: 554-560.

6. Ryan CF, Love LL, Peat D, Fleetham JA, Lowe AA. Mandibular advancement oral appliance therapy for obstructive sleep apnoea: effect on awake calibre of the velopharynx. Thorax 1999; 54: 972-977.

7. Kawauchi Y, Oshima T, Suzuki S, Saitoh Y, Toyooka H. Advancement of the mandible facilitates nasal breathing in human subjects sedated with midazolam. Can J Anaesth 2000; 47: 215-219.

8. Lorino AM, Maza M, d'Ortho MP, Coste A, Harf A, Lorino H. Effects of mandibular advancement on respiratory resistance. Eur Respir J 2000; 16: 928-932.

9. Pae EK, Lowe AA, Fleetham JA. A role of pharyngeal length in obstructive sleep apnea patients. Am J Orthod Dentfac Orthop 1997; 111: 12-17.

10. Hiyama S, Ono T, Ishiwata Y, Kuroda T. Supine cephalometric study on sleep-related changes in upper-airway structures in normal subjects. Sleep 2000; 23: 783-790.
11. Solow B, Tallgren A. Head posture and craniofacial morphology. Am J Phys Anthropol 1976; 44: 417-436.

12. Hair JF, Anderson RE, Tatham RL, Black WC. Multivariate data analysis. 5th Edn. New Jersey, Prentice-Hall, 1998; pp. 33-86.

13. Tsuiki S, Ono T, Kuroda T. Mandibular advancement modulates respiratory-related genioglossus electromyographic activity. Sleep Breath 2000; 4: 53-57.

14. Clark GT, Arand D, Chung E, Tong D. Effect of anterior mandibular positioning on obstructive sleep apnea. Am Rev Respir Dis 1993; 147: 624-629.

15. Eveloff SE, Rosenberg CL, Carlisle CC, Millman RP Efficacy of a Herbst mandibular advancement device in obstructive sleep apnea. Am J Respir Crit Care Med 1994; 149: 905-909.

16. Henke KG, Frantz DE, Kuna ST. An oral elastic mandibular advancement device for obstructive sleep apnea. Am J Respir Crit Care Med 2000; 161: 420-425.

17. Kato J, Isono S, Tanaka A, et al. Dose-dependent effects of mandibular advancement on pharyngeal mechanics and nocturnal oxygenation in patients with sleep-disordered breathing. Chest 2000; 117: 1065-1072.

18. Kuna ST, Remmers JE. Anatomy and physiology of upper airway obstruction. In: Kryger MH, Roth T, Dement WC, eds. Principles and Practice of Sleep Medicine. 3rd Edn. Philadelphia, WB Saunders, 2000; pp. 840-858.

19. Mehta A, Qian J, Petocz P, Darendeliler MA, Cistulli PA. A randomized, controlled study of a mandibular advancement splint for obstructive sleep apnoea. Am J Respir Crit Care Med 2001; 163: 1457-1461.

20. Morrison DL, Launois SH, Isono S, Feroah TR, Whitelaw WA, Remmers JE. Pharyngeal narrowing and closing pressure in patients with obstructive sleep apnea. Am Rev Respir Dis 1993; 148: 606-611.

21. Isono S, Tanaka A, Sho Y, Konno A, Nishino A. Advancement of the mandible improves velopharyngeal airway patency. J Appl Physiol 1995; 79: 2132-2138.

22. Tsuiki S, Almeida FR, Bhalla PS, Lowe AA, Fleetham JA. Supine-dependent changes in upper airway size in awake obstructive sleep apnea patients. Sleep Breath 2003; 7: 43-49.

23. Horner RL, Guz A. Some factors affecting the maintenance of upper airway patency in man. Respir Med 1991; 85: Suppl. A, 27-30.

24. Shome B, Wang LP, Santare MH, Prasad AK, Szeri AZ, Roberts D. Modeling of airflow in the pharynx with application to sleep apnea. J Biomech Eng 1998; 120: 416422.

25. Schwab R. Upper airway imaging. Sleep Disorders. In: Strollo PJ Jr, Sanders MH, eds. Clinics in Chest Medicine. Volume 19, number 1. Philadelphia, WB Saunders, 1998; pp. 33-54.

26. Hiyama S, Tsuiki S, Ono T, Kuroda T, Ohyama K. Effects of mandibular advancement on supine airway size in normal subjects during sleep. Sleep 2003; 26: 440-445.

27. Leiter JC. Upper airway shape. Is it important in the pathogenesis of obstructive sleep apnea? Am J Respir Crit Care Med 1996; 153: 894-898.

28. Lowe AA, Fleetham JA. Two- and three-dimensional analyses of tongue, airway, and soft palate size. In: Norton ML, Brown ACD, eds. Atlas of the Difficult Airway. St Louis, Mosby, 1991; pp. 74-82.

29. Ferguson KA, Ono T, Lowe AA, Al-Majed S, Love LL, Fleetham JA. A short-term controlled trial of an adjustable oral appliance for the treatment of mild to moderate obstructive sleep apnoea. Thorax 1997; 52: 362-368.

30. Quinlan M, Salman S, Swift DL, Wagner HN Jr, Proctor DF. Measurement of mucociliary function in man. Am Rev Respir Dis 1969; 99: 624-629. 